MATHEMATICS OF COMPUTATION

Volume 79, Number 272, October 2010, Pages 2103-2115

S 0025-5718(10)02370-7

Article electronically published on April 29, 2010

\title{
TWO-POINT TAYLOR EXPANSIONS AND ONE-DIMENSIONAL BOUNDARY VALUE PROBLEMS
}

\author{
JOSÉ L. LÓPEZ AND ESTER PÉREZ SINUSÍA
}

\begin{abstract}
We consider second-order linear differential equations $\varphi(x) y^{\prime \prime}+$ $f(x) y^{\prime}+g(x) y=h(x)$ in the interval $(-1,1)$ with Dirichlet, Neumann or mixed Dirichlet-Neumann boundary conditions. We consider $\varphi(x), f(x), g(x)$ and $h(x)$ analytic in a Cassini disk with foci at $x= \pm 1$ containing the interval $(-1,1)$. The two-point Taylor expansion of the solution $y(x)$ at the extreme points \pm 1 is used to give a criterion for the existence and uniqueness of solution of the boundary value problem. This method is constructive and provides the two-point Taylor approximation of the solution(s) when it exists.
\end{abstract}

\section{INTRODUCTION}

Consider the second-order linear differential equation $\varphi(x) y^{\prime \prime}+f(x) y^{\prime}+g(x) y=$ $h(x)$ in a real interval $(a, b)$ with $\varphi(x)>0$. By means of an affine change of independent variable, we can transform the interval $(a, b)$ into the interval $(-1,1)$ and, without loss of generality, consider the boundary value problem

$$
\left\{\begin{array}{l}
\varphi(x) y^{\prime \prime}+f(x) y^{\prime}+g(x) y=h(x) \text { in }(-1,1), \\
B\left(\begin{array}{c}
y(-1) \\
y(1) \\
y^{\prime}(-1) \\
y^{\prime}(1)
\end{array}\right)=\left(\begin{array}{l}
\alpha \\
\beta
\end{array}\right),
\end{array}\right.
$$

with $\alpha, \beta \in \mathbb{R}$ and $B$ a $2 \times 4$ matrix of rank 2 which defines the (Dirichlet, Neumann or mixed) boundary conditions. A standard theorem for the existence and uniqueness of solution of (1.1) is based on the knowledge of the two-dimensional linear space of solutions of the equation $\varphi(x) y^{\prime \prime}+f(x) y^{\prime}+g(x) y=0$ 2, Chap.4, Sec. 1]. When $\varphi, f, g$ and $h$ are constants or in some other particular situations, it is possible to find a general solution of the equation (sometimes via the Green's function [2, Chap. 4], [8, Chaps. 1 and 3]). But, in general situations, this is not possible, and that standard criterion for the existence and uniqueness of solution of (1.1) is not practical. Another well-known criterion for the existence and uniqueness of solution of (1.1) is based on the Lax-Milgram theorem when (1.1) is an

Received by the editor May 5, 2009.

2010 Mathematics Subject Classification. Primary 34A25, 34B05, 41A58.

Key words and phrases. Second-order linear differential equations, boundary value problem, Frobenius method, two-point Taylor expansions.

The Ministerio de Ciencia y Tecnología (REF. MTM2007-63772) and the Gobierno de Navarra (Res. 228/2008) are acknowledged by their financial support. The Department of Theoretical Physics of the University of Zaragoza is also acknowledged by its hospitality.

(c)2010 American Mathematical Society Reverts to public domain 28 years from publication 
elliptic problem [3. In any case, the determination of the existence and uniqueness of solution of (1.1) requires a non-systematic detailed study of the problem, for example, the study of the eigenvalue problem associated to (1.1) [2, Chap. 4, Sec. 2], [8, Chap. 7].

When $\varphi, f, g$ and $h$ are analytic in a disk with center at $x=0$ and containing the interval $[-1,1]$ with $\varphi>0$ in $[-1,1]$, we may consider the initial value problem

$$
\left\{\begin{array}{l}
\varphi(x) y^{\prime \prime}+f(x) y^{\prime}+g(x) y=h(x), \quad x \in(-1,1) \\
y(0)=y_{0}, \quad y^{\prime}(0)=y_{0}^{\prime}
\end{array}\right.
$$

with $y_{0}, y_{0}^{\prime} \in \mathbb{R}$. Using the Frobenius method we can approximate the solution of this problem by its Taylor polynomial of degree $N$ at $x=0, y_{N}(x)=\sum_{n=0}^{N} c_{k} x^{k}$, where the coefficients $c_{k}$ are affine functions of $c_{0}=y_{0}$ and $c_{1}=y_{0}^{\prime}$. By imposing the boundary conditions given in (1.1) over $y_{N}(x)$, we obtain an algebraic linear system for $y_{0}$ and $y_{0}^{\prime}$. The existence and uniqueness of solution of this algebraic linear system gives us information about the existence and uniqueness of solution of (1.1). This procedure, although theoretically possible, has a difficult practical implementation, since the data of the problem are given at $x= \pm 1$, not at $x=0$ [1, 7]. Moreover, when $\varphi, f, g$ or $h$ have a singularity close to the interval $[-1,1]$ or $\varphi$ vanishes at a point close to the interval $[-1,1]$, the above mentioned disk does not contain the interval $[-1,1]$ and the Taylor series of the solution $y(x)$ does not converge $\forall x \in[-1,1]$. In this case we can use a Taylor expansion of the solution at several points along the interval $[-1,1]$ and match these expansions at intersecting disks [6, Sec. 7]. In this way, we obtain an approximation of the solution of (1.1) in the form of a piecewise polynomial in several subintervals of $[-1,1]$. But this approximation is not uniform in the whole interval $[-1,1]$, and the matching of the expansions translates into numerical errors.

The purpose of this paper is to improve this idea using, not the standard Taylor expansion in the associated initial value problem (1.2), but a two-point Taylor expansion at the extreme points $x= \pm 1$ (see [4]) directly in the boundary value problem (1.1). In [5] we have shown that, when $\varphi, f, g$ and $h$ are analytic in a region containing the interval $[-1,1]$, a two-point Taylor expansion of the solution $y(x)$ at the two extreme points of the interval \pm 1 is useful to approximate the solution of the boundary value problem. The convergence region for that two-point Taylor expansion is a Cassini disk (see Figure 1) that avoids the possible singularities of the coefficient functions more efficiently than the standard Taylor disk 5 .

In [5] we have assumed that problem (1.1) has a unique solution and then we have proposed several algorithms to approximate that solution. The purpose of this paper is different. We want to use the two-point Taylor expansion of the solution $y(x)$ to give an existence and uniqueness criterion based on the data of the problem, not based on the knowledge of the general solution of the differential equation, and it does not require a deep functional analysis. This criterion is systematic and is derived in Section 2. Moreover, our method is constructive and provides a systematic algorithm to approximate the solution(s) of (1.1) (when it exists). Section 3 considers the particular case of polynomial coefficients. Section 4 contains some final remarks. 


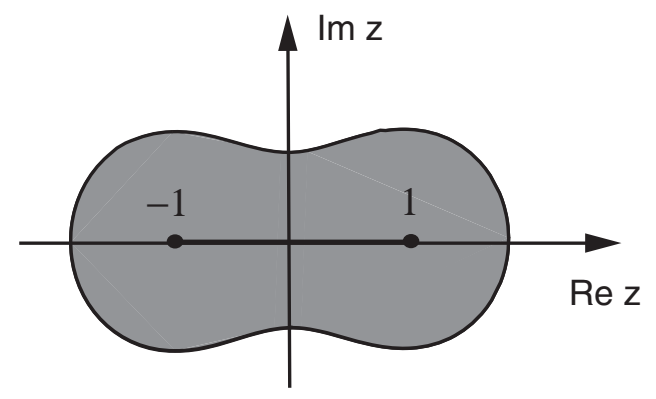

Figure 1. The Cassini disk $\mathcal{D}_{r}=\left\{z \in \mathbb{C}|| z^{2}-1 \mid<r\right\}$ with foci at $z= \pm 1$ and radius $r>1$ contains the real interval $[-1,1]$.

\section{EXISTENCE AND UNIQUENESS CRITERION}

Assume that the coefficient functions $\varphi, f, g, h$ in (1.1) are analytic in the Cassini disk $\mathcal{D}_{r}=\left\{z \in \mathbb{C}|| z^{2}-1 \mid<r\right\}$ with foci at $z= \pm 1$ and Cassini's radius $r$, with $r>1$ and $\varphi(x)>0$ in $\mathcal{D}_{r}$ (see [4]). The requirement $r>1$ assures that the interval $(-1,1)$ is contained in the Cassini disk $\mathcal{D}_{r}$ (see Figure 1).

Then, we have that any solution of the differential equation in (1.1) can be represented in the form of a two-point Taylor expansion at the base points $x= \pm 1$ [5]

$$
y(x)=\sum_{n=0}^{\infty}\left[a_{n}+b_{n} x\right]\left(x^{2}-1\right)^{n}, \quad x \in(-1,1),
$$

with $a_{n}$ and $b_{n}$ two sequences of complex numbers related to the derivatives of $y(x)$ at $x= \pm 1$ [4. This series is absolutely and uniformly convergent in the interval $[-1,1]$. We also have [5]:

$$
\begin{aligned}
y^{\prime}(x)= & \sum_{k=0}^{\infty}\left\{\left[(2 k+1) b_{k}+2(k+1) b_{k+1}\right]+2(k+1) a_{k+1} x\right\}\left(x^{2}-1\right)^{k}, \\
y^{\prime \prime}(x)= & \sum_{k=0}^{\infty} 2(k+1)\left\{\left[(2 k+1) a_{k+1}+2(k+2) a_{k+2}\right]\right. \\
& \left.+\left[(2 k+3) b_{k+1}+2(k+2) b_{k+2}\right] x\right\}\left(x^{2}-1\right)^{k} .
\end{aligned}
$$

From (2.1) and (2.2) we have

$$
\left(\begin{array}{c}
y(-1) \\
y(1) \\
y^{\prime}(-1) \\
y^{\prime}(1)
\end{array}\right)=T\left(\begin{array}{l}
a_{0} \\
b_{0} \\
a_{1} \\
b_{1}
\end{array}\right),
$$

where $T$ is the rank- 4 matrix

$$
T=\left(\begin{array}{cccc}
1 & -1 & 0 & 0 \\
1 & 1 & 0 & 0 \\
0 & 1 & -2 & 2 \\
0 & 1 & 2 & 2
\end{array}\right) .
$$

(The first four coefficients $a_{0}, b_{0}, a_{1}, b_{1}$ of the two-point Taylor expansion (2.1) are related to $y(-1), y(1), y^{\prime}(-1), y^{\prime}(1)$ through the matrix $T^{-1}$.) Then, the boundary 
value problem (1.1) reads

$$
\left\{\begin{array}{l}
\varphi(x) y^{\prime \prime}+f(x) y^{\prime}+g(x) y=h(x) \text { in }(-1,1), \\
\tilde{R}\left(\begin{array}{l}
a_{0} \\
b_{0} \\
a_{1} \\
b_{1}
\end{array}\right)=\left(\begin{array}{l}
\alpha \\
\beta
\end{array}\right)
\end{array}\right.
$$

with $\tilde{R}=B T$. Denote by $R_{i, j}, i=1,2, j=1,2,3,4$, the entries of this matrix $\tilde{R}$.

On the other hand, as it happens in the standard Frobenius method for initial value problems, the coefficients $a_{n}$ and $b_{n}$ of the two-point Taylor expansion (2.1) of the solution $y(x)$ of the differential equation in (2.5) satisfy a system of recurrences of the form [5]

$$
\begin{aligned}
a_{n} & =\sum_{k=0}^{n-1}\left[A_{n, k} a_{k}+B_{n, k} b_{k}\right]+E_{n}, \quad n=2,3,4, \ldots, \\
b_{n} & =\sum_{k=0}^{n-1}\left[C_{n, k} a_{k}+D_{n, k} b_{k}\right]+F_{n}, \quad n=2,3,4, \ldots,
\end{aligned}
$$

where the coefficients $A_{n, k}, B_{n, k}, E_{n}, C_{n, k}, D_{n, k}$ and $F_{n}$ depend on the two-point Taylor coefficients of $\varphi, f, g$ and $h$ at $x= \pm 1$. In general, as in the standard Frobenius method, the computation of the coefficients $a_{n}$ and $b_{n}$ involve the previous coefficients $a_{0}, b_{0}, \ldots, a_{n-1}$ and $b_{n-1}$. But when $\varphi, f, g$ and $h$ are polynomials, these recurrences are of finite order (say $s$ ) and the computation of the coefficients $a_{n}$ and $b_{n}$ only involve the previous $2 s$ coefficients $a_{n-s}, b_{n-s}, \ldots, a_{n-1}$ and $b_{n-1}$. We illustrate this situation with the following example.

Example 2.1. Consider the boundary value problem

$$
\left\{\begin{array}{l}
\left(x^{2}+1\right)^{2} y^{\prime \prime}+3 x\left(x^{2}+1\right) y^{\prime}+2 y=0 \quad \text { in }(-1,1), \\
y(-1)=y(1)=\frac{1}{2}
\end{array}\right.
$$

We have $\varphi(x)=\left(x^{2}+1\right)^{2}, f(x)=3 x\left(x^{2}+1\right), g(x)=2$ and $h=0$. The function $\varphi$ is nonvanishing in the Cassini disk $\mathcal{D}_{r}$ with foci at $x= \pm 1$ and $[-1,1] \subset \mathcal{D}_{r}$ for any $1<r<2$ (see [5]). The two-point Taylor expansions of the coefficient functions are finite:

$$
\begin{gathered}
\varphi(x)=[4+0 \cdot x]+[4+0 \cdot x]\left(x^{2}-1\right)+[1+0 \cdot x]\left(x^{2}-1\right)^{2}, \\
f(x)=[0+6 \cdot x]+[0+3 \cdot x]\left(x^{2}-1\right), \quad g(x)=[2+0 \cdot x],
\end{gathered}
$$

and then, the recursions (2.6) are of order $s=3$. For $n=2,3,4, \ldots$ and $a_{-1}=$ $b_{-1}=0$,

$$
\begin{aligned}
8 n(n-1) a_{n}=-2(n-1)[(2 n-3)+2(3 n-4)] a_{n-1} \\
-\left[\left(4(n-2)^{2}+n-1\right)+2(n-2)(3 n-5)\right] a_{n-2}-2(n-2)(n-3) a_{n-3}, \\
16 n(n-1) b_{n}=-4(n-1)[(2 n-1)+6(n-1)] b_{n-1} \\
-\left[(4 n-5)(2 n-3)+2+3(2 n-3)^{2}\right] b_{n-2}-\left[4(n-2)^{2}-1\right] b_{n-3} .
\end{aligned}
$$


As in the Frobenius method, the order of the recurrences is at least two, that is, $s \geq 2$. But, as a difference with the Frobenius method where we only have one recurrence for the sequence of standard Taylor coefficients, here we have a system of two recurrences. In the standard Frobenius method designed for an initial value problem of the form:

$$
\left\{\begin{array}{l}
\varphi(x) y^{\prime \prime}+f(x) y^{\prime}+g(x) y=h(x), \\
y(0)=c_{0}, \quad y^{\prime}(0)=c_{1}
\end{array}\right.
$$

we look for a solution of the form $y(x)=\sum_{n=0}^{\infty} c_{n} x^{n}$. Then, the computation of the coefficients $c_{n}$ for $n \geq 2$ only requires the initial seed $c_{0}$ and $c_{1}$ that are data of the problem.

The situation is different for the boundary value problem (2.5) when we look for a solution of the form (2.1). Since, in this case, we have a system of two recurrences instead of only one recurrence, the computation of the coefficients $a_{n}, b_{n}$ for $n \geq 2$ requires the initial seed $a_{0}, a_{1}, b_{0}$ and $b_{1}$. This does not mean that the linear space of solutions of the differential equation in (2.5) has dimension four; this space has of course dimension two. It is happening here that, apart from the two-dimensional linear space $S$ of (true) solutions of the differential equation in (2.5), there is a bigger space of formal solutions $W$ defined by

$W:=\left\{y(x)=\sum_{n=0}^{\infty}\left[a_{n}+b_{n} x\right]\left(x^{2}-1\right)^{n} \mid a_{n}, b_{n}\right.$ given in (2.6) $\left., a_{0}, b_{0}, a_{1}, b_{1} \in \mathbb{R}\right\}$.

Formally, all the two-point series in $W$ are solutions of the differential equation in (2.5). But not all of them are convergent, only a subset: the two-dimensional linear space $S$ of (true) solutions that may be identified as

$$
S=\left\{y \in W \mid \sum_{n=0}^{\infty}\left[a_{n}+b_{n} x\right]\left(x^{2}-1\right)^{n} \text { is uniformly convergent in }[-1,1]\right\} .
$$

In order to give a more practical characterization of $S$, we must find a linear system of two independent equations for the parameters $a_{0}, b_{0}, a_{1}, b_{1}$. This is the purpose of the remainder of the section.

For a fixed $m \in \mathbb{N}, m \geq 2$, we define the vector

$$
v_{n}:=\left(a_{n+2-m}, b_{n+2-m}, a_{n+3-m}, b_{n+3-m}, \ldots, a_{n}, b_{n}, a_{n+1}, b_{n+1}\right) \in \mathbb{R}^{2 m}
$$

with $a_{-k}=b_{-k}=0$ for $k \in \mathbb{N}$. In particular we have

$$
v_{m-2}=\left(a_{0}, b_{0}, a_{1}, b_{1}, \ldots, a_{m-1}, b_{m-1}\right) \quad \text { and } \quad v_{0}=\left(0,0, \ldots, 0,0, a_{0}, b_{0}, a_{1}, b_{1}\right) .
$$

For $n=0,1,2, \ldots, m-2$, define the $(2 m) \times(2 m)$ matrix

$$
M_{n}:=\left(\begin{array}{ccccccccc}
0 & 0 & 1 & 0 & 0 & 0 & 0 & \ldots & 0 \\
0 & 0 & 0 & 1 & 0 & 0 & 0 & \ldots & 0 \\
\ldots & \ldots & \ldots & \ldots & \ldots & \ldots & \ldots & \ldots & \ldots \\
\ldots & \ldots & \ldots & \ldots & \ldots & \ldots & \ldots & \ldots & \ldots \\
0 & 0 & 0 & 0 & 0 & \ldots & 0 & 1 & 0 \\
0 & 0 & 0 & 0 & 0 & \ldots & 0 & 0 & 1 \\
0 & \ldots & 0 & A_{n+2,0} & B_{n+2,0} & \ldots & \ldots & A_{n+2, n+1} & B_{n+2, n+1} \\
0 & \ldots & 0 & C_{n+2,0} & D_{n+2,0} & \ldots & \ldots & C_{n+2, n+1} & D_{n+2, n+1}
\end{array}\right) .
$$


The only non-null elements of this matrix are those corresponding to the entries $m_{i, i+2}=1, i=1,2,3, \ldots, 2 m-2$, and to the entries $m_{2 m-1, k}, m_{2 m, k}$, $k=0,1,2, \ldots, n+1$. In particular, we have

$$
M_{0}=\left(\begin{array}{ccccccccc}
0 & 0 & 1 & 0 & 0 & 0 & 0 & \ldots & 0 \\
0 & 0 & 0 & 1 & 0 & 0 & 0 & \ldots & 0 \\
\ldots & \ldots & \ldots & \ldots & \ldots & \ldots & \ldots & \ldots & \ldots \\
\ldots & \ldots & \ldots & \ldots & \ldots & \ldots & \ldots & \ldots & \ldots \\
0 & 0 & 0 & 0 & 0 & \ldots & 0 & 1 & 0 \\
0 & 0 & 0 & 0 & 0 & \ldots & 0 & 0 & 1 \\
0 & 0 & \ldots & 0 & 0 & A_{2,0} & B_{2,0} & A_{2,1} & B_{2,1} \\
0 & 0 & \ldots & 0 & 0 & C_{2,0} & D_{2,0} & C_{2,1} & D_{2,1}
\end{array}\right)
$$

and

$$
M_{m-2}=\left(\begin{array}{ccccccccc}
0 & 0 & 1 & 0 & 0 & 0 & 0 & \ldots & 0 \\
0 & 0 & 0 & 1 & 0 & 0 & 0 & \ldots & 0 \\
\ldots & \ldots & \ldots & \ldots & \ldots & \ldots & \ldots & \ldots & \ldots \\
\ldots & \ldots & \ldots & \ldots & \ldots & \ldots & \ldots & \ldots & \ldots \\
0 & 0 & 0 & 0 & 0 & \ldots & 0 & 1 & 0 \\
0 & 0 & 0 & 0 & 0 & \ldots & 0 & 0 & 1 \\
A_{m, 0} & B_{m, 0} & A_{m, 1} & B_{m, 1} & \ldots & \ldots & \ldots & A_{m, m-1} & B_{m, m-1} \\
C_{m, 0} & D_{m, 0} & C_{m, 1} & D_{m, 1} & \ldots & \ldots & \ldots & C_{m, m-1} & D_{m, m-1}
\end{array}\right) .
$$

We also need, for $n=0,1,2, \ldots, m-2$, to define the vector

$$
c_{n}:=\left(0,0, \ldots, 0,0, E_{n+2}, F_{n+2}\right) \in \mathbb{R}^{2 m} .
$$

Then, the system of recurrences (2.6) can be written in a matrix form. For $n=$ $1,2,3, \ldots, m-1$ we have

$$
v_{n}=M_{n-1} v_{n-1}+c_{n-1} .
$$

To find the solution of this linear recurrence for the vector $v_{n}$, we recurrently define the following matrices:

$$
\begin{aligned}
& \mathcal{M}_{0}=M_{0}, \quad \mathcal{M}_{n}=M_{n} \mathcal{M}_{n-1}, \quad n=1,2,3, \ldots, m-2, \\
& \mathcal{C}_{0}=c_{0}, \quad \mathcal{C}_{n}=M_{n} \mathcal{C}_{n-1}+c_{n}, \quad n=1,2,3, \ldots, m-2,
\end{aligned}
$$

or

$$
\begin{gathered}
\mathcal{M}_{n}=\prod_{k=0}^{n} M_{n-k} \\
\mathcal{C}_{n}=c_{n}+\sum_{k=0}^{n-1}\left[M_{n} \cdot M_{n-1} \cdots M_{k+1}\right] c_{k} .
\end{gathered}
$$

Then, we find

$$
v_{m-1}=\mathcal{M}_{m-2} v_{0}+\mathcal{C}_{m-2}
$$


or, in an extended form,

$$
\begin{aligned}
& \left(\begin{array}{c}
\star \\
\star \\
\cdot \\
\cdot \\
\cdot \\
\star \\
a_{m} \\
b_{m}
\end{array}\right)=\left(\begin{array}{c}
\star \\
\star \\
\cdot \\
\cdot \\
\cdot \\
\star \\
\mathcal{B}_{2 m-1} \\
\mathcal{B}_{2 m}
\end{array}\right)
\end{aligned}
$$

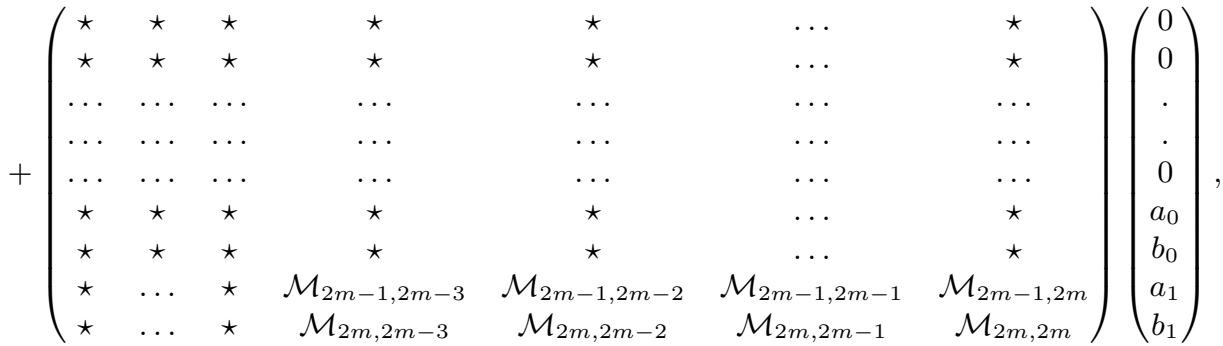

where the $\star$ 's denote real (unspecified) numbers. The two-point Taylor series of $S$ converge in $[-1,1]$ if they converge at $x=0$, and they converge at $x=0$ if and only if $\lim _{m \rightarrow \infty}\left(a_{m}, b_{m}\right)=(0,0)$. Then, taking the limit $m \rightarrow \infty$ into the above equation we find

$$
\left(\begin{array}{c}
\star \\
\star \\
\cdot \\
\cdot \\
\cdot \\
\star \\
\star \\
0 \\
0
\end{array}\right)=\left(\begin{array}{ccccccccc}
\star & \star & \star & \star & \star & \star & \star & \ldots & \star \\
\star & \star & \star & \star & \star & \star & \star & \ldots & \star \\
\ldots & \ldots & \ldots & \ldots & \ldots & \ldots & \ldots & \ldots & \ldots \\
\ldots & \ldots & \ldots & \ldots & \ldots & \ldots & \ldots & \ldots & \ldots \\
\ldots & \ldots & \ldots & \ldots & \ldots & \ldots & \ldots & \ldots & \ldots \\
\star & \star & \star & \star & \star & \star & \star & \ldots & \star \\
\star & \star & \star & \star & \star & \star & \star & \ldots & \star \\
\star & \star & \ldots & \star & \star & R_{3,1} & R_{3,2} & R_{3,3} & R_{3,4} \\
\star & \star & \ldots & \star & \star & R_{4,1} & R_{4,2} & R_{4,3} & R_{4,4}
\end{array}\right)\left(\begin{array}{c}
0 \\
0 \\
\cdot \\
\cdot \\
0 \\
a_{0} \\
b_{0} \\
a_{1} \\
b_{1}
\end{array}\right)+\left(\begin{array}{c}
\star \\
\star \\
\cdot \\
\cdot \\
\cdot \\
\star \\
\star \\
-\gamma \\
-\delta
\end{array}\right),
$$

where

$$
\begin{aligned}
R_{4-i, j} & :=\lim _{m \rightarrow \infty} \mathcal{M}_{2 m-i, 2 m+j-4}, \quad i=0,1, j=1,2,3,4, \\
-\gamma & :=\lim _{m \rightarrow \infty} \mathcal{B}_{2 m-1}, \quad-\delta:=\lim _{m \rightarrow \infty} \mathcal{B}_{2 m} .
\end{aligned}
$$

Then, the two equations that we were looking for are

$$
\left(\begin{array}{llll}
R_{3,1} & R_{3,2} & R_{3,3} & R_{3,4} \\
R_{4,1} & R_{4,2} & R_{4,3} & R_{4,4}
\end{array}\right)\left(\begin{array}{l}
a_{0} \\
b_{0} \\
a_{1} \\
b_{1}
\end{array}\right)=\left(\begin{array}{l}
\gamma \\
\delta
\end{array}\right) .
$$

These two equations are linearly independent and reduce the number of free parameters from four to two. The proof of the linear independence of (2.11) is as follows. Consider the initial value problem

$$
\left\{\begin{array}{l}
\varphi(x) y^{\prime \prime}+f(x) y^{\prime}+g(x) y=h(x), \\
y(-1)=y_{-1}, \quad y^{\prime}(-1)=y_{-1}^{\prime}
\end{array}\right.
$$


with $y_{-1}, y_{-1}^{\prime} \in \mathbb{R}$ and seek a solution in the form (2.1). The coefficients $a_{0}, b_{0}, a_{1}, b_{1}$ of the two-point Taylor solution of this initial value problem are solutions of the linear system (2.11) and also of the two linear equations imposed by the initial conditions $y(-1)=y_{-1}$ and $y^{\prime}(-1)=y_{-1}^{\prime}$ :

$$
\left(\begin{array}{cccc}
1 & -1 & 0 & 0 \\
0 & 1 & -2 & 2 \\
R_{3,1} & R_{3,2} & R_{3,3} & R_{3,4} \\
R_{4,1} & R_{4,2} & R_{4,3} & R_{4,4}
\end{array}\right)\left(\begin{array}{l}
a_{0} \\
b_{0} \\
a_{1} \\
b_{1}
\end{array}\right)=\left(\begin{array}{c}
y_{-1} \\
y_{-1}^{\prime} \\
\gamma \\
\delta
\end{array}\right)
$$

If the rank of the coefficient matrix of this system was not four, then the initial value problem (2.12) would have more than one solution or no solution. Therefore, the two equations in (2.11) are linearly independent.

One precision must be done at this point. It is possible that some or all the row coefficients $\mathcal{M}_{2 m-i, 2 m+j-4}, i=0,1, j=1,2,3,4$, in the matrix $\mathcal{M}$ have no limit when $m \rightarrow \infty$. But then, at least, there must be two subsequences of row coefficients $\mathcal{M}_{2 m-i, 2 m+j-4}, i=0,1, j=1,2,3,4$, in the matrix $\mathcal{M}$ having as a limit two independent rows $\left(R_{3,1}, R_{3,2}, R_{3,3}, R_{3,4}\right)$, $\left(R_{4,1}, R_{4,2}, R_{4,3}, R_{4,4}\right)$. Otherwise, the initial value problem (2.12) would have more than one solution or no solution. Then, the limits of (2.10) must be understood as limits of certain subsequences.

Joining the two equations in (2.11) with the two algebraic equations provided by the boundary conditions in (2.5), we find the following linear system of four equations and four unknowns:

$$
\left(\begin{array}{llll}
R_{1,1} & R_{1,2} & R_{1,3} & R_{1,4} \\
R_{2,1} & R_{2,2} & R_{2,3} & R_{2,4} \\
R_{3,1} & R_{3,2} & R_{3,3} & R_{3,4} \\
R_{4,1} & R_{4,2} & R_{4,3} & R_{4,4}
\end{array}\right)\left(\begin{array}{l}
a_{0} \\
b_{0} \\
a_{1} \\
b_{1}
\end{array}\right)=\left(\begin{array}{c}
\alpha \\
\beta \\
\gamma \\
\delta
\end{array}\right)
$$

The two first equations of this system are obtained from the boundary conditions, whereas the other two equations are obtained from the differential equation in (2.5).

At this point, we can formulate the following existence and uniqueness criterion for the boundary value problem (1.1):

The existence and uniqueness of solution of the boundary value problem (1.1) is equivalent to the existence and uniqueness of solution of the linear system (2.13). More precisely,

- When the linear system (2.13) has a unique solution $\left(a_{0}, b_{0}, a_{1}, b_{1}\right)$, the boundary value problem (1.1) has a unique solution given by (2.1) and (2.6) with $\left(a_{0}, b_{0}, a_{1}, b_{1}\right)$ the solution of (2.13).

- When the linear system (2.13) has infinite solutions (one- or two of the parameters $a_{0}, b_{0}, a_{1}, b_{1}$ are free), the boundary value problem (1.1) has a one- or two-parametric family of solutions given by (2.1) and (2.6) with $\left(a_{0}, b_{0}, a_{1}, b_{1}\right)$ the solution of (2.13).

- When the linear system (2.13) has no solution, the boundary value problem (1.1) has no solution.

The two first rows of the system (2.13) are independent. The two last rows are also independent. This means that the dimension of the space of solutions of (1.1) is at most two.

If we denote by $R$ the coefficient matrix of the system (2.13), $x:=\left(a_{0}, b_{0}, a_{1}, b_{1}\right)$ the vector of unknowns and $\Lambda:=(\alpha, \beta, \gamma, \delta)$ the vector of independent terms, 2.13) 
can be written as the system $R x=\Lambda$. In practice, the exact computation of the limits (2.10) is impossible, and we must approximate them in the form

$$
\begin{aligned}
R_{4-i, j} & \simeq \mathcal{M}_{2 m-i, 2 m+j-4}, \quad i=0,1, j=1,2,3,4, \\
-\gamma & \simeq \mathcal{B}_{2 m-1}, \quad-\delta \simeq \mathcal{B}_{2 m}
\end{aligned}
$$

for a large enough value of $m$. This means that, in practice, we work with an approximate system $R_{m} x_{m}=\Lambda_{m}$ instead of the system $R x=\Lambda$. Then, the values of the coefficients $x_{m}$ obtained from $R_{m} x_{m}=\Lambda_{m}$ are approximations of the exact coefficients $a_{0}, b_{0}, a_{1}, b_{1}$.

Also, in practice, we must apply the above existence and uniqueness criterion for the solution of (1.1) using the approximate linear system $R_{m} x_{m}=\Lambda_{m}$ instead of the exact system $R x=\Lambda$. Nevertheless, the conclusions about existence and uniqueness are the same unless the system $R x=\Lambda$ is not well conditioned. In this case, the ranks of the coefficient matrix $R_{m}$ and/or of the augmented matrix $\left(R_{m} \mid \Lambda_{m}\right)$ sensibly depend on the precision in the computation of the approximate limits (2.14).

\section{Polynomial coefficients}

When the coefficient functions $\varphi, f, g$ and $h$ are polynomials, we can somehow simplify the formulation of the above existence and uniqueness criterion. In general, as we have seen in the previous section, the computation of the coefficients $\left(a_{n}, b_{n}\right)$ requires a matrix of size $(2 m) \times(2 m)$ with $m \geq n$. This means that we need matrices of increasing size to compute the coefficients. In the case of polynomial coefficients, the situation is different. The recurrences (2.6) are of constant order $s$ independent of $n$ and the computation of the coefficients $a_{n}$ and $b_{n}$ involve only the previous $2 s$ coefficients $a_{n-s}, b_{n-s}, \ldots, a_{n-1}$ and $b_{n-1}$. Thus, in this case, we do not need matrices of increasing size, but matrices of constant size $(2 s) \times(2 s)$.

The recurrence system (2.6) for polynomial coefficients is of the form

$$
\begin{aligned}
a_{n} & =\sum_{k=n-s}^{n-1}\left[A_{n, k} a_{k}+B_{n, k} b_{k}\right]+E_{n}, \\
b_{n} & =\sum_{k=n-s}^{n-1}\left[C_{n, k} a_{k}+D_{n, k} b_{k}\right]+F_{n}
\end{aligned}
$$

for a certain $s \in \mathbb{N}, n=0,1,2, \ldots$, with $a_{-k}=b_{-k}=0, n, k \in \mathbb{N}$. The discussion is identical to the one in the previous section, but we can eliminate the restriction $n \leq m$. Moreover, we can simplify the computations, because now the size of the matrices $M_{n}$ does not depend on $n$. We can now define the matrices $M_{n}$ of fixed size $(2 s) \times(2 s)$ in the form

$$
M_{n}:=\left(\begin{array}{ccccccccc}
0 & 0 & 1 & 0 & 0 & 0 & 0 & \ldots & 0 \\
0 & 0 & 0 & 1 & 0 & 0 & 0 & \ldots & \ldots \\
\ldots & \ldots & \ldots & \ldots & \ldots & \ldots & \ldots & \ldots & \ldots \\
\ldots & \ldots & \ldots & \ldots & \ldots & \ldots & \ldots & \ldots & 0 \\
0 & 0 & 0 & 0 & 0 & \ldots & 0 & 1 & 0 \\
A_{n+2, n+2-s} & B_{n+2, n+2-s} & \ldots & A_{n+2,0} & B_{n+2,0} & \ldots & \ldots & A_{n+2, n+1} & B_{n+2, n+1} \\
C_{n+2, n+2-s} & D_{n+2, n+2-s} & \ldots & C_{n+2,0} & D_{n+2,0} & \ldots & \ldots & C_{n+2, n+1} & D_{n+2, n+1}
\end{array}\right)
$$

instead of the form (2.9), with $A_{n,-k}=B_{n,-k}=C_{n,-k}=D_{n,-k}=0$ for $k \in \mathbb{N}$. The computation of the system (2.13) is identical. The only difference is that now the matrices $\mathcal{M}_{m}$ are of size $(2 s) \times(2 s) \forall m \in \mathbb{N}$ and the vectors $\mathcal{C}_{m} \in \mathbb{R}^{2 s} \forall m \in \mathbb{N}$. 


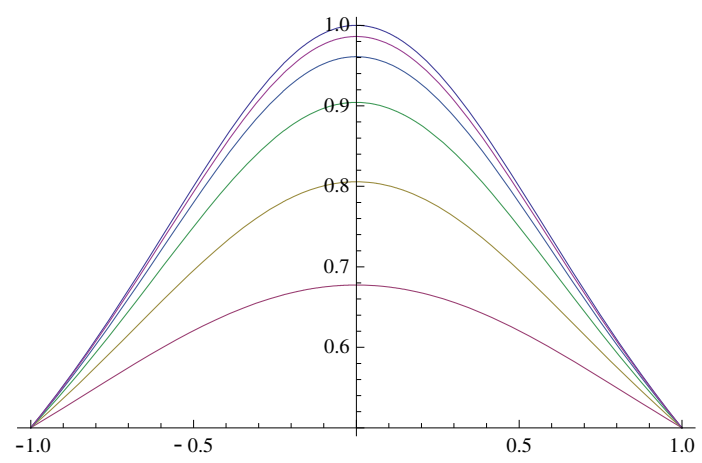

Figure 2. Plot of the exact solution $y(x)=\left(x^{2}+1\right)^{-1}$ (curve on the top) of (2.7) and the approximations $y_{n}(x)$ for $n=0,1,2, \ldots, 8$.

Example 3.1. As an example of a boundary value problem with polynomial coefficients, we consider the problem defined in (2.7). The recurrences (2.8) are of order $s=3$ and may be written in the form $v_{n+1}=M_{n} v_{n}$ with $v_{n}=\left(a_{n-1}, b_{n-1}, a_{n}, b_{n}\right.$, $\left.a_{n+1}, b_{n+1}\right)$ and

$$
M_{n}=\left(\begin{array}{cccccc}
0 & 0 & 1 & 0 & 0 & 0 \\
0 & 0 & 0 & 1 & 0 & 0 \\
0 & 0 & 0 & 0 & 1 & 0 \\
0 & 0 & 0 & 0 & 0 & 1 \\
\frac{n(1-n)}{4(n+1)(n+2)} & 0 & -\frac{10 n^{2}+3 n+1}{8(n+1)(n+2)} & 0 & -\frac{8 n+5}{4(n+2)} & 0 \\
0 & \frac{1-4 n^{2}}{16(n+1)(n+2)} & 0 & -\frac{10 n^{2}+11 n+4}{8(n+1)(n+2)} & 0 & -\frac{8 n+9}{4(n+2)}
\end{array}\right) .
$$

For example, for $m=10$, the linear system $R_{m} x_{m}=\Lambda_{m}$ reads

$$
\left(\begin{array}{cccc}
1 & -1 & 0 & 0 \\
1 & 1 & 0 & 0 \\
-0.0159524 & 0 & -0.033858 & 0 \\
0 & -1.54795 & 0 & -9.84517
\end{array}\right)\left(\begin{array}{c}
a_{0} \\
b_{0} \\
a_{1} \\
b_{1}
\end{array}\right)=\left(\begin{array}{c}
0.5 \\
0.5 \\
0 \\
0
\end{array}\right) .
$$

This system has a unique solution $\left(a_{0}, b_{0}, a_{1}, b_{1}\right)=(0.5,0,-0.235579,0)$, and then the boundary value problem (2.7) has a unique solution that can be approximated by the two-point Taylor polynomials

$$
y_{n}(x)=\sum_{k=0}^{n}\left[a_{k}+b_{k} x\right]\left(x^{2}-1\right)^{k}, \quad n=0,1,2, \ldots .
$$

For example, we obtain the following polynomials for $n=2,4$ and 6 :

$$
\begin{aligned}
& y_{2}(x)=0.5-0.235579\left(x^{2}-1\right)+0.115987\left(x^{2}-1\right)^{2} \\
& y_{4}(x)=y_{2}(x)-0.0569417\left(x^{2}-1\right)^{3}+0.0277667\left(x^{2}-1\right)^{4} \\
& y_{6}(x)=y_{4}(x)-0.0133721\left(x^{2}-1\right)^{5}+0.00629436\left(x^{2}-1\right)^{6} .
\end{aligned}
$$

Figure 2 illustrates the accuracy of the approximations $y_{n}(x)$ in (3.3) obtained for $m=10$ and $n=0,1,2, \ldots, 8$.

The following example shows the application of the above criterion to a boundary value problem depending on parameters. 
Example 3.2. Consider the boundary value problem

$$
\left\{\begin{array}{l}
y^{\prime \prime}-2 x y^{\prime}-2 y=2 a, \quad \text { in }(-1,1) \\
y(-1)+y(1)=c \\
y^{\prime}(-1)+b y^{\prime}(1)=0
\end{array}\right.
$$

with $a, b$ and $c$ real parameters. We have $\varphi(x)=1, f(x)=-2 x, g(x)=-2$ and $h(x)=2 a$. The two-point Taylor expansions of these coefficient functions are finite: $\varphi(x)=[1+0 \cdot x], \quad f(x)=[0-2 \cdot x], \quad g(x)=[-2+0 \cdot x], \quad h(x)=[2 a+0 \cdot x]$, and then, the recursions (2.6) are of order $s=2$. For $n=2,3,4, \ldots$

$$
\begin{aligned}
2 n(n-1) a_{n} & =-(n-1)(2 n-5) a_{n-1}+(2 n-3) a_{n-2}+\frac{a}{2} \delta_{n-2,0}, \\
2 n b_{n} & =-(2 n-3) b_{n-1}+2 b_{n-2} .
\end{aligned}
$$

The recurrences (3.6) may be written in the form $v_{n+1}=M_{n} v_{n}+s \delta_{n, 0}$ with $v_{n}=\left(a_{n}, b_{n}, a_{n+1}, b_{n+1}\right)$ and

$$
M_{n}=\left(\begin{array}{cccc}
0 & 0 & 1 & 0 \\
0 & 0 & 0 & 1 \\
\frac{1+2 n}{2(n+1)(n+2)} & 0 & -\frac{2 n-1}{2(n+2)} & 0 \\
0 & \frac{1}{n+2} & 0 & -\frac{2 n+1}{2(n+2)}
\end{array}\right), \quad s=\left(\begin{array}{c}
0 \\
0 \\
a / 4 \\
0
\end{array}\right) .
$$

For $m=10$, the linear system $R_{m} x_{m}=\Lambda_{m}$ is given by

$$
\left(\begin{array}{cccc}
2 & 0 & 0 & 0 \\
0 & b+1 & 2(b-1) & 2(b+1) \\
0.016618 & 0 & -0.0166177 & 0 \\
0 & 0.186893 & 0 & -0.250427
\end{array}\right)\left(\begin{array}{c}
a_{0} \\
b_{0} \\
a_{1} \\
b_{1}
\end{array}\right)=\left(\begin{array}{c}
c \\
0 \\
-0.016618 a \\
0
\end{array}\right)
$$

Now applying the criterion of Section 2, the existence and uniqueness of solution of (3.5) is equivalent to the existence and uniqueness of solution of (3.7) that, in this example, depends on the values of the parameters $a, b$ and $c$ :

- If $b \neq-1$, the system (3.7) has a unique solution and then (3.5) has a unique solution.

- If $b=-1$ and $2 a+c=0$, the system (3.7) has infinite solutions and then (3.5) has infinite solutions.

- If $b=-1$ and $2 a+c \neq 0$, the system (3.7) has no solution and then (3.5) has no solution.

We next observe that the results obtained with this criterion coincide with the ones provided by the standard criterion. The general solution of the differential equation given in (3.5) is

$$
y(x)=c_{1} e^{x^{2}} \operatorname{erf}(x)+c_{2} e^{x^{2}}-a .
$$

The standard criterion of existence and uniqueness of solution depends on the existence of real numbers $c_{1}$ and $c_{2}$ that makes $y(x)$ compatible with the boundary conditions in (3.5). That is, it depends on the existence of a solution of the linear system

$$
\left(\begin{array}{cc}
{[1+e \sqrt{\pi} \operatorname{erf}(1)](b+1)} & 2 e \\
\sqrt{\pi} & (b-1) e
\end{array}\right)\left(\begin{array}{l}
c_{1} \\
c_{2}
\end{array}\right)=\left(\begin{array}{c}
2 a+c \\
0
\end{array}\right) .
$$



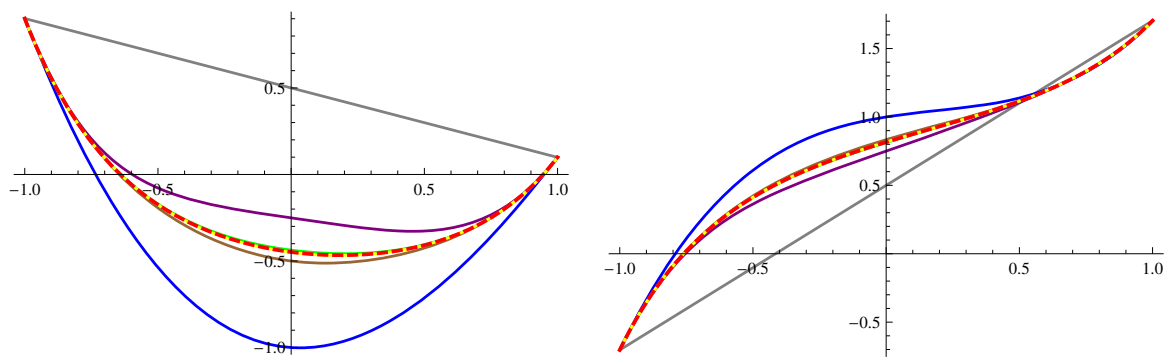

Figure 3. Plot of the exact solution $y(x)=$ $\frac{\sqrt{\pi}(1-b)(2 a+c)}{2(1+b)(1+e \sqrt{\pi} \operatorname{erf}(1))} e^{x^{2}} \operatorname{erf}(x)+\frac{2 a+c}{2 e} e^{x^{2}}-a$ (dashed) of (3.5) for $a=c=1, b=2$ (first graph), $a=c=-1, b=-2$ (second graph) and the approximations $y_{n}(x)$ for $n=0,1,2, \ldots, 5$.

It is easy to check that the study of the existence and uniqueness of solution of (3.8) provides the same conclusions as the ones obtained with our criterion.

Figure 3 illustrates the accuracy of the approximations $y_{n}(x)$ in (3.3) obtained for $m=10, n=0,1,2, \ldots, 5$ and different values of the parameters $a, b$ and $c$.

\section{Final REMARKS}

At the end of Section 2 we have given a straightforward and systematic criterion for the existence and uniqueness of solution of boundary value problems for second-order linear differential equations (1.1) when the coefficients of the differential equation are analytic functions inside a Cassini disk containing the domain of the differential equation. The criterion is very simple and establishes that the existence and uniqueness of solution of the boundary value problem (1.1) is equivalent to the existence and uniqueness of solution of the algebraic linear system $R x=\Lambda$ given in (2.13). Half of the entrances of the system (2.13) are defined by the limits (2.10), whose exact computation is, in general, very difficult. In practice, the entrances of the system (2.13) must be computed approximately in the form (2.14), and then the solution of the system $R x=\Lambda, x=\left(a_{0}, b_{0}, a_{1}, b_{1}\right)$ is approximated by the solution of the system $R_{m} x_{m}=\Lambda_{m}$. Also, in practice, we must apply the above existence and uniqueness criterion for the solution of (1.1) using the approximate linear system $R_{m} x_{m}=\Lambda_{m}$ instead of the exact linear system $R x=\Lambda$. Nevertheless, the conclusions about the existence and uniqueness of solution are exact unless the system $R x=\Lambda$ is not well-conditioned. In this case, the ranks of the coefficient matrix $R_{m}$ and/or of the augmented matrix $\left(R_{m} \mid \Lambda_{m}\right)$ sensibly depend on the precision in the computation of the approximate limits (2.14).

Formally, the criterion proposed in this paper is similar to the standard criterion, based on the knowledge of the space of solutions: both criteria relate the existence and uniqueness of solution of the boundary value problem (1.1) to the existence and uniqueness of a solution of an algebraic linear system. As a difference with that standard criterion, our criterion does not require the knowledge of the general solution of the differential equation. This qualitative difference is very important when the general solution of the equation is not known. In this case, the standard criterion is not useful, whereas our criterion can always be applied (except in the case of bad conditioning as discussed before). 
The use of the alternative theorem for boundary value problems [8, Chap. 3, Sec. 4] does not help to simplify our criterion. This theorem asserts that problem (1.1) has a unique solution if and only if a certain associated completely homogeneous problem has a unique solution. The application of our technique to that completely homogeneous problem does not suppose any substantial simplification; the only difference is that for that homogeneous problem, the algebraic linear system (2.13) is homogeneous. But moreover, when that homogeneous linear system has many solutions, we would still have to decide between many solutions or no solution for the problem (1.1) by computing the inner product of the independent term of (1.1) with a system of independent solutions of the associated homogeneous problem [8, p. 229].

\section{REFERENCES}

1. C. Kesan, Taylor polynomial solutions of linear differential equations, Appl. Math. Comp. 142(1) (2003), 155-165. MR1978253

2. A. C. King, J. Billingham and S. R. Otto, Differential Equations. Linear, Nonlinear, Ordinary, Partial, Annals of Mathematics Studies. Princeton Univ. Press 33 (1954), 167-190. MR1996393 (2004d:34001)

3. P. D. Lax and A. N. Milgram, Parabolic Equations. Contributions to the theory of partial differential equations, Cambridge Univ. Press, New York, 2003. MR0067317 (16:709b)

4. J. L. López and Nico M. Temme, Two-point Taylor expansions of analytic functions, Stud. Appl. Math. 109(4) (2002), 297-311. MR1934653 (2003g:30005)

5. J. L. López, E. Pérez Sinusía and N. Temme, Multi-point Taylor approximations in onedimensional linear boundary value problems, Appl. Math. Comput. 207 (2009), 519-527. MR2489122

6. A. B. Olde Daalhuis and F. W. J. Olver, On the asymptotic and numerical solution of linear ordinary differential equations, SIAM Rev. 40(3) (1998), 463-495. MR.1642803 (99g:34117)

7. M. Sezer, A method for the approximate solution of the second-order linear differential equations in terms of Taylor polynomials, Int. J. Math. Edu. Sci. Technol. 27 (1996), 821-834.

8. I. Stakgold, Green's functions and boundary value problems, Wiley and Sons, New York, 1988.

Departamento de Ingeniería Matemática e Informática, Universidad Pública de Navarra, 31006-PAmplona, Spain

E-mail address: j1.lopez@unavarra.es

Departamento de Matemática Aplicada, Universidad de Zaragoza, 50018-Zaragoza, SPAIN

E-mail address: ester.perez@unizar.es 